\title{
The effect of predator avoidance and travel time delay on the stability of predator-prey metacommunities
}

\author{
Yuxiang Zhang \\ Department of Mathematics and Statistics \\ University of Ottawa, Ottawa, ON, K1N 6N5 \\ and \\ Frithjof Lutscher \\ Department of Mathematics and Statistics, and Department of Biology \\ University of Ottawa, Ottawa, ON, K1N 6N5 \\ and \\ Frédéric Guichard \\ Department of Biology \\ McGill University, Montreal, QC H3A 1B1
}

\begin{abstract}
The stability conditions for an isolated specialist predator-prey community are fairly well understood. The spatial coupling of several such systems through dispersal of individuals can generate new dynamic behavior that is not yet completely understood. Many factors are known to be stabilizing or neutral, e.g. random dispersal or time delays, others may induce instabilities in some cases but not others, e.g. densitydependent movement. We study the combination of two stabilizing mechanisms in a
\end{abstract}


two-patch Rosenzweig-MacArthur model with a novel density-dependent movement term. Specifically, we assume that prey move between patches according to their perceived predation risk, and we include travel time between patches as a time delay. We show that the combination of mechanisms may be destabilizing even though each mechanism by itself is stabilizing. Our results show that a detailed knowledge of mechanisms and their temporal scales is necessary to correctly predict the stability of a metacommunity.

\section{Introduction}

The movement of individuals, and the constraints imposed to movement over natural landscapes are key features of community dynamics that affect persistence and resilience of populations, species coexistence, and ecosystem function (Nathan and Giuggioli, 2013). Ecological implications of movement can be predicted from simplistic assumptions of passive and instantaneous movement between locations. However, more complex patterns of movement associated with species traits (life-history, behavior) and/or with environmental heterogeneity can have major ecological consequences. Most importantly, this interplay betwen species traits and the environment is greatly impacted by the management of exploited ecosystems. While simulation studies have incorporated complex behaviors (McCauley et al., 1993) and movement patterns in complex landscapes (Cuddington and Yodzis, 2002; Ruokolainen et al., 2011), there is still an important gap between general ecological theory of spatial dynamics, and our understanding of complex mechanisms and patterns of movement in natural landscapes. Here we study the interaction between the time of movement across landscapes and behavioral drivers of movement to avoid predators. We integrate these features of landscapes and traits into a simple discrete-habitat model that extends current ecological theory. In particular, we study the stability of predatorprey dynamics in terms of the time scales of movement controled by both behavior (predator avoidance) and life-history (dispersal and migration) in relation to movement time across landscapes. 
Trophic interactions between predators and their prey constitute a model system for the study of dynamical stability. Rosenzweig and MacArthur (1963) showed how such a simple ecological community can switch from stable equilibrium to stable limit cycles in response to increased carrying capacity of the prey (i.e. the paradox of enrichment). This simple model has generated a large body of literature on the role of individual movement as a stabilizing mechanism in simple two-patch systems, as well as spatially explicit models (Briggs and Hoopes, 2004; Cantrell and Cosner, 1996; Hauzy et al., 2010; Jansen, 2001; Ramanantoanina et al., 2011). The most general analyses of such models result from the assumption of random and instantanuous movement of individuals between homogeneous locations. Many studies have been able to relax this assumption and include more complex behavioral mechanisms of movement and habitat heterogeneity such as taxis (Sapoukhina et al., 2003; Chakraborty et al., 2007), density-dependent movement (Abrams and Ruokolainen, 2011), and travel time (Klepac et al., 2007). Mostly, these mechanisms have been studied separately or in simulation studies with specific parameter values. An integration into a general ecological theory is still missing.

Natural landscapes can be described as a matrix of various habitat types, each with its own impact on individuals (Goodwin and Fahrig, 2002). In its simplest form, landscapes are represented as two or more suitable patches where a population can establish, and surrounded by a landscape matrix, only allowing for movement between suitable habitats. This complex structure can be simplified by only considering the time of travel between suitable habitats instead of assuming instantanuous transfer of individuals (Klepac et al., 2007). However, when movement is associated with life-history traits such as dispersal of larvae or propagules, movement is assumed to be short compared to individual lifespan, which limits its study to arbitrarily low values. Alternatively, when movement is triggered by mechanisms operating over much shorter time sale such as behavior, movement time can be scaled to that of behavioral responses. For example, the role of predator avoidance resulting in a non-consumptive response of prey to predator density can be formulated as density- dependent movement, and has been well studied using models and experiments (Abdllaoui et al., 
2007; Mchich et al., 2005, 2007). It has been generalised to behavioral response of prey to total expected fitness, and to evolutionary dynamics of the traits mediating the response (Abrams, 2007). This behavioral response to predator density means that movement changes much faster than the expected lifespan of individuals, and can thus interact with travel time between habitats. Here we study community persistence and stability in response to the interaction between non-consumptive prey response to predators and travel time of prey between two discrete habitats. We more specifically present and analyze a two-patch extension of the Rosenzweig-MacArthur predator-prey model with a travel time (time delay) in both density-dependent and density-independent movement of the prey. We derive the time scales of densityindependent and density-dependent movement relative to travel time that predict both stabilizing and destabilizing effects of movement on the spatially-structured community.

In the following section, we present the details of our model and show that as long as movement is instantaneous, the inclusion of predator-dependent dispersal does not alter the stability of the coexistence steady state. When movement is not instantaneous, then an unstable coexistence equilibrium can be stabilized by densityindependent dispersal (Section 3.1). In addition, stable coexistence can be destabilized by predator-induced dispersal (Section 3.2). Throughout the manuscript, we will use the terms "coexistence (steady) state" and "coexistence equilibrium" interchangeably. When coexistence occurs in the form of a stable limit cycle, we will explicitly say so.

\section{The Model}

A minimal model that allows us to study how predator-dependent dispersal and travel-time delay interact to affect the stability of a multi-patch predator-prey system tracks the density of prey $\left(H_{i}\right)$ and predator $\left(P_{i}\right)$ on two patches $(i=1,2)$ through time, $t$. On each patch, we assume MacArthur-Rosenzweig dynamics: the prey grows logistically in the absence of the predator and is consumed by the predator according 
to a type-II functional response; the predator decays exponentially in the absence of prey. In the absence of predators, prey move randomly between the two patches with exponentially distributed waiting time. When predators are present, prey also leave a patch according to their perceived predation risk, which is proportional to the per capita of prey predation rate in the functional response. In particular, the perceived predation risk increases with the number of predators and decreases with the number of prey. Previous work had considered movement rates dependent on predator density only (e.g. Abdllaoui et al. (2007)) or on fitness (Abrams, 2007). The total rate of movement between the two patches is a linear interpolation between random movement and predation avoidance. The time $(\eta)$ that it takes an individual to travel from one patch to the other enters the equations in the form of a delay. For simplicity, we assume that the two patches are identical and that the predator does not move between patches. We write our model as

$$
\begin{aligned}
\frac{d H_{1}}{d t}= & r H_{1}\left(1-\frac{H_{1}}{K}\right)-\frac{a c H_{1} P_{1}}{b+H_{1}}-D\left(\alpha \frac{\rho P_{1}}{b+H_{1}}+(1-\alpha)\right) H_{1} \\
& +D\left(\alpha \frac{\rho P_{2}(t-\eta)}{b+H_{2}(t-\eta)}+(1-\alpha)\right) H_{2}(t-\eta), \\
\frac{d P_{1}}{d t}= & \frac{a H_{1} P_{1}}{b+H_{1}}-m P_{1}, \\
\frac{d H_{2}}{d t}= & r H_{2}\left(1-\frac{H_{2}}{K}\right)-\frac{a c H_{2} P_{2}}{b+H_{2}}-D\left(\alpha \frac{\rho P_{2}}{b+H_{2}}+(1-\alpha)\right) H_{2} \\
& +D\left(\alpha \frac{\rho P_{1}(t-\eta)}{b+H_{1}(t-\eta)}+(1-\alpha)\right) H_{1}(t-\eta), \\
\frac{d P_{2}}{d t}= & \frac{a H_{2} P_{2}}{b+H_{2}}-m P_{2},
\end{aligned}
$$

where $D>0$ is the movement rate and $0 \leq \alpha \leq 1$ denotes the contribution of predator-induced dispersal to the total dispersal rate. The prey growth rate and carrying capacity are $r$ and $K$. Parameter $a$ is the maximum ingestion rate, $b$ is the half saturation constant and $c$ is the inverse of the yield. The predator death rate is denoted by $m$, and $\rho$ is a scaling factor proportional to $c$. We have several characteristic times in our model, we focus on a few only. The inverse of the ingestion 


\begin{tabular}{|c|l|c|}
\hline Symbol & Description & dimension \\
\hline$H_{i}$ & prey density on patch $i$ & {$[\mathrm{H}]=\frac{\# \text { prey }}{\text { space }}$} \\
$P_{i}$ & predator density on patch $i$ & {$[\mathrm{P}]=\frac{\# \text { predator }}{\text { space }}$} \\
$t$ & time & time \\
$\eta$ & travel time between patches & time \\
\hline$r$ & prey growth rate & $\frac{1}{\text { time }}$ \\
$K$ & carrying capacity & {$[H]$} \\
$a$ & maximum ingestion rate & $\frac{1}{\text { time }}$ \\
$b$ & half-saturation constant for predation & $\frac{\#}{\text { space }}$ \\
$c$ & inverse of yield & $\frac{[H]}{[P]}$ \\
$m$ & predator mortality & $\frac{1}{\text { time }}$ \\
\hline$D$ & between-patch movement rate & $\frac{1}{\text { time }}$ \\
$\alpha$ & fraction of density-dependent movement & - \\
$\rho$ & conversion factor & {$[H] /[P]$} \\
\hline
\end{tabular}

Table 1: Summary of all parameters, their meaning and their dimensions.

rate is the mean time between subsequent meals for the predator, which reflects the time between subsequent successful captures. We call $1 / a$ the mean time to capture is $1 / a$. The characteristic time of dispersal consists of the mean residence time on a patch, $1 / D$, and the (fixed) travel time between patches, $\eta$. All parameters in our model are summarized in Table 1

We reduce the number of parameters by passing to non-dimensional quantities $H_{i}=b h_{i}, P_{i}=\frac{b r}{a c} p_{i}, t=\frac{1}{a} s, \eta=\frac{1}{a} \tau, k=\frac{K}{b}, \gamma=\frac{\rho r}{a c}, d=\frac{D}{a}, \epsilon=\frac{r}{a}, \mu=\frac{m}{a}$. The resulting dimensionless system of equations is as follows. 


$$
\begin{aligned}
\frac{d h_{1}}{d s}= & \epsilon h_{1}\left(1-\frac{h_{1}}{k}-\frac{p_{1}}{1+h_{1}}\right)-d\left(\alpha \gamma \frac{p_{1}}{1+h_{1}}+(1-\alpha)\right) h_{1} \\
& +d\left(\alpha \gamma \frac{p_{2}(s-\tau)}{1+h_{2}(s-\tau)}+(1-\alpha)\right) h_{2}(s-\tau), \\
\frac{d p_{1}}{d s}= & \frac{h_{1} p_{1}}{1+h_{1}}-\mu p_{1}, \\
\frac{d h_{2}}{d s}= & \epsilon h_{2}\left(1-\frac{h_{2}}{k}-\frac{p_{2}}{1+h_{2}}\right)-d\left(\alpha \gamma \frac{p_{2}}{1+h_{2}}+(1-\alpha)\right) h_{2} \\
& +d\left(\alpha \gamma \frac{p_{1}(s-\tau)}{1+h_{1}(s-\tau)}+(1-\alpha)\right) h_{1}(s-\tau), \\
\frac{d p_{2}}{d s}= & \frac{h_{2} p_{2}}{1+h_{2}}-\mu p_{2} .
\end{aligned}
$$

In the absence of dispersal, the model consists of two independent copies of the MacArthur-Rosenzweig system in the form

$$
\begin{aligned}
\frac{d h_{i}}{d s} & =\epsilon h_{i}\left(1-\frac{h_{i}}{k}-\frac{p_{i}}{1+h_{i}}\right) \\
\frac{d p_{i}}{d s} & =\frac{h_{i} p_{i}}{1+h_{i}}-\mu p_{i} .
\end{aligned}
$$

This well-known system has up to three steady states: the trivial state $(0,0)$, the predator-extinction state $(k, 0)$, and the coexistence state $\left(h^{*}, p^{*}\right)$, where $h^{*}=\frac{\mu}{1-\mu}$ and $p^{*}=\left(1-\frac{h^{*}}{k}\right)\left(1+h^{*}\right)$, provided that $0<h^{*}<k$. The predator-extinction state is stable precisely when $h^{*}>k$. When $(k-1) / 2<h^{*}<k$, the coexistence equilibrium is globally stable, but when $0<h^{*}<(k-1) / 2$ the coexistence state is unstable and a unique globally stable limit cycle exists (see e.g. Kot (2001)).

The three equilibria from the single-patch model translate into three corresponding equilibria the two-patch model since the assumption of equal patches gives a symmetric system. These are $(0,0,0,0),(k, 0, k, 0)$, and $\left(h^{*}, p^{*}, h^{*}, p^{*}\right)$, respectively. In addition, since predators do not move between patches, there can be two boundary equilibria $\left(\bar{h}, 0, h^{*}, \bar{p}\right)$ and $\left(h^{*}, \bar{p}, \bar{h}, 0\right)$ for some positive numbers $\bar{h}, \bar{p}$. We focus the analysis on the stability of the symmetric positive coexistence equilibrium $\left(h^{*}, p^{*}, h^{*}, p^{*}\right)$. 
When movement between patches is instantaneous, i.e. $\tau=0$, our model is a special case of the model in (Hauzy et al., 2010). Those authors showed that then the coexistence equilibrium $\left(h^{*}, p^{*}, h^{*}, p^{*}\right)$ of $(2.2)$ is stable if and only if the coexistence equilibrium $\left(h^{*}, p^{*}\right)$ of $(2.3)$ is stable. For convenience of the reader, we give the proof of this fact in Appendix A. In other words, if dispersal between patches is instantaneous, then predation-induced prey movement does not affect the stability of the coexistence steady state. From now on, we study the case when dispersal is not instantaneous.

\section{Stability of the coexistence equilibrium}

When travel time is included in the model $(\tau>0)$, the stability analysis of the coexistence steady state becomes more difficult and reveals destabilizing mechanisms. Throughout this section we shall assume $0<h^{*}<k$. We linearize system (2.2) at the coexistence state by setting $h_{i}=h^{*}+u_{i}$ and $p_{i}=p^{*}+v_{i}$, for small perturbations $u_{i}, v_{i}$. Keeping only the linear terms in the Taylor expansion of the right hand side of (2.2) leads to the transcendental eigenvalue problem

$$
\begin{aligned}
\lambda u_{1}= & {\left[\epsilon\left(1-\frac{2 h^{*}}{k}-\frac{p^{*}}{\left(1+h^{*}\right)^{2}}\right)-d\left(\alpha \gamma \frac{p^{*}}{\left(1+h^{*}\right)^{2}}+1-\alpha\right)\right] u_{1}-(\epsilon+d \alpha \gamma) \mu v_{1} } \\
& +d\left(\alpha \gamma \frac{p^{*}}{\left(1+h^{*}\right)^{2}}+1-\alpha\right) e^{-\lambda \tau} u_{2}+d \alpha \gamma \mu e^{-\lambda \tau} v_{2} \\
\lambda v_{1}= & \frac{p^{*}}{\left(1+h^{*}\right)^{2}} u_{1}, \\
\lambda u_{2}= & {\left[\epsilon\left(1-\frac{2 h^{*}}{k}-\frac{p^{*}}{\left(1+h^{*}\right)^{2}}\right)-d\left(\alpha \gamma \frac{p^{*}}{\left(1+h^{*}\right)^{2}}+1-\alpha\right)\right] u_{2}-(\epsilon+d \alpha \gamma) \mu v_{2} } \\
& +d\left(\alpha \gamma \frac{p^{*}}{\left(1+h^{*}\right)^{2}}+1-\alpha\right) e^{-\lambda \tau} u_{1}+d \alpha \gamma \mu e^{-\lambda \tau} v_{1} \\
\lambda v_{2}= & \frac{p^{*}}{\left(1+h^{*}\right)^{2}} u_{2}
\end{aligned}
$$

for nonzero $\left(u_{1}, v_{1}, u_{2}, v_{2}\right)$. The coexistence state is stable if all solutions $\lambda$ have negative real part.

System (3.1) admits a nonzero solution if and only if the determinant of the corresponding coefficient matrix is zero. Due to the symmetry of the system, this 
coefficient matrix can be expressed in the form

$$
J=\left(\begin{array}{cc}
J_{1}-\lambda I & J_{2} \\
J_{2} & J_{1}-\lambda I
\end{array}\right),
$$

where $I$ is the $2 \times 2$ identity matrix. Since $J$ is a block-circulant matrix with $2 \times 2$ blocks, its determinant is given by $\operatorname{det} J=\operatorname{det}\left(J_{1}-\lambda I+J_{2}\right) \cdot \operatorname{det}\left(J_{1}-\lambda I-J_{2}\right)$. Therefore, a nonzero solution of (3.1) exists if and only if $\operatorname{det}\left(J_{1}-\lambda I+J_{2}\right)=0$ or $\operatorname{det}\left(J_{1}-\lambda I-J_{2}\right)=0$.

These two conditions are equivalent to

$$
L(\lambda)=-R(\lambda) \quad \text { or } \quad L(\lambda)=R(\lambda),
$$

where

$$
\begin{aligned}
L(\lambda)= & \lambda^{2}-\lambda\left[\epsilon\left(1-\frac{2 h^{*}}{k}-\frac{p^{*}}{\left(1+h^{*}\right)^{2}}\right)-d\left(\frac{\alpha \gamma p^{*}}{\left(1+h^{*}\right)^{2}}+1-\alpha\right)\right] \\
& +\frac{\mu p^{*}}{\left(1+h^{*}\right)^{2}}(\epsilon+d \alpha \gamma), \\
R(\lambda)= & {\left[d \lambda\left(\frac{\alpha \gamma p^{*}}{\left(1+h^{*}\right)^{2}}+1-\alpha\right)+\frac{\mu p^{*}}{\left(1+h^{*}\right)^{2}} d \alpha \gamma\right] e^{-\lambda \tau} . }
\end{aligned}
$$

Then coexistence equilibrium of (2.2) is stable if and only if all characteristic roots of (3.2) have negative real parts; otherwise, it is unstable. To analyze (3.2), we rely on a result from Cooke and Grossman (1982) that states that a change of stability in our system can occur only if roots of (3.2) appear on the imaginary axis. For the convenience of the reader, we quote and explain this result in Appendix B.

Since we know the stability conditions for the coexistence state when $d, \alpha, \tau$ are zero, we can ask whether those three parameters affect stability by studying how zeros of (3.2) on the imaginary axis depend on those three parameters. For simplicity, we first treat the case $\alpha=0$ separately in the next section and then move on to the general case.

\subsection{Density-independent dispersal $(\alpha=0)$}

When $\alpha=0$, the characteristic equations (3.2) become

$$
\lambda^{2}-\lambda(\epsilon A-d)+\epsilon B= \pm d \lambda e^{-\lambda \tau}
$$


where we denoted

$$
A=1-\frac{2 h^{*}}{k}-\frac{p^{*}}{\left(1+h^{*}\right)^{2}}, \quad B=\frac{\mu p^{*}}{\left(1+h^{*}\right)^{2}}>0 .
$$

The expression $\epsilon A$ is precisely the trace of the community matrix of the RosenzweigMacArthur model (2.3) at the coexistence steady state. In particular, this steady state is stable on the single patch if and only if $A<0$.

Since zero is not a root of (3.3), and since complex roots appear in pairs, we look for roots $\lambda=\mathrm{i} y$, with $y>0$. Splitting the equations in (3.3) into real and imaginary part gives the system

$$
\left\{\begin{aligned}
-y^{2}+\epsilon B & = \pm d y \sin (y \tau) \\
-(\epsilon A-d) & = \pm d \cos (y \tau)
\end{aligned}\right.
$$

Case 1: $A<0$

When $A<0$, the steady state on the single-patch model is stable. The second condition in (3.5) becomes

$$
|\cos (y \tau)|=1-\frac{\epsilon A}{d}>1
$$

and this condition is impossible to satisfy. Hence, no solutions of (3.3) can appear on the imaginary axis for any $d, \tau$. Since the coexistence state is locally asymptotically stable for $d=0, \tau=0$, it is also locally asymptotically stable for all $d, \tau>0$. In particular, density-independent dispersal and travel time delays cannot destabilize a stable coexistence state in the absence of density-dependent dispersal.

Case 2: $A>0$

When the positive equilibrium is unstable for the single-patch model $(2.3)(A>0)$, we ask whether and under which conditions dispersal and/or travel time delay can stabilize the system. As above, such a change in stability can only happen on a critical curve in the $(\tau, d)$-plane on which the characteristic equations (3.3) have a purely imaginary root. 
When the dispersal rate is small, no such roots can appear, and the coexistence equilibrium cannot be stabilized by dispersal and/or travel time delay. More precisely, when $0 \leq d<\epsilon A / 2$, then the second condition in (3.3) becomes

$$
|\cos (y \tau)|=\frac{\epsilon A}{d}-1>1
$$

which is impossible. Since the eigenvalues for $\tau=0$ have positive real parts, they will have positive real parts for all $\tau>0$, provided that $0 \leq d<\epsilon A / 2$. In dimensional parameters, this condition reads

$$
D<\frac{r m}{2 a}\left[1-\frac{b}{K} \frac{a+m}{a-m}\right]
$$

When the dispersal rate is large enough, the coexistence equilibrium can be stabilized for some intermediate values of $\tau$, but the relationship between $d$ and $\tau$ where stabilization occurs is complex. To understand this relationship in detail, we summarize some of the analysis of the characteristic equations (3.3), using the results by Cooke and Grossman (1982). For a fixed value $d>\epsilon A>0$ and $\tau=0$, equation (3.3) with the plus-sign has two solutions with negative real parts whereas the equation with the minus-sign has two solutions with positive real part, representing the fact that the coexistence state is unstable for the single patch model. As $\tau$ increases from zero, the number of solutions of equation (3.3) with positive real part increases or decreases by two at certain values of $\tau$ that can be computed explicitly. If the number of solutions with positive real part of (3.3) with the minus-sign decreases before the number of such solutions to (3.3) with the plus-sign increases, then the coexistence steady state can be stabilized. We now give a detailed description of these threshold values for $\tau$.

We begin with equation (3.3) and the plus-sign. According to Cooke and Grossman (1982), there are two sequences $\left\{\tau_{n, 1}\right\}$ and $\left\{\tau_{n, 2}\right\}$ defined by the relationships

$$
\tau_{n, 1}=\frac{\theta_{1}+2 n \pi}{y_{1}}, \tau_{n, 2}=\frac{\theta_{2}+2 n \pi}{y_{2}}, n=0,1,2 \ldots
$$




$$
\begin{aligned}
& \theta_{1}=\cos ^{-1}(\epsilon A / d-1) \in(\pi / 2, \pi) \\
& \theta_{2}=\cos ^{-1}(\epsilon A / d-1) \in(\pi, 3 \pi / 2) \\
& y_{1}=\frac{1}{2}\left(d^{2}-(\epsilon A-d)^{2}\right)^{\frac{1}{2}}+\frac{1}{2}\left(d^{2}-(\epsilon A-d)^{2}+4 B \epsilon\right)^{\frac{1}{2}}>0 \\
& y_{2}=-\frac{1}{2}\left(d^{2}-(\epsilon A-d)^{2}\right)^{\frac{1}{2}}+\frac{1}{2}\left(d^{2}-(\epsilon A-d)^{2}+4 B \epsilon\right)^{\frac{1}{2}}>0 .
\end{aligned}
$$

We obtain these expressions from (3.5) by squaring, adding the equations and solving for $y$. These sequences are increasing and nested so that $\tau_{0,1}<\tau_{0,2}<\tau_{1,1}<\tau_{1,2}<\ldots$ As $\tau$ increases, the multiplicity of roots with positive real part increases by two when $\tau$ passes through $\tau_{n, 1}$ and decreases by two when $\tau$ passes through $\tau_{n, 2}$ until some finite $\tau_{N, 1}$, after which the multiplicity will remain positive.

Similarly, for equation (3.3) and the minus-sign there are increasing interlaced sequences $\left\{\tau_{n, 3}\right\}$ and $\left\{\tau_{n, 4}\right\}$ defined by

$$
\begin{aligned}
\tau_{n, 3} & =\frac{\theta_{3}+2 n \pi}{y_{2}}, \tau_{n, 4}=\frac{\theta_{4}+2 n \pi}{y_{1}}, n=0,1,2 \ldots, \\
\theta_{3} & =\cos ^{-1}(1-\epsilon A / d) \in(0, \pi / 2) \\
\theta_{4} & =\cos ^{-1}(1-\epsilon A / d) \in(3 \pi / 2,2 \pi)
\end{aligned}
$$

such that total multiplicity of the roots with positive real parts decreases by two as $\tau$ increases and passes through any value of $\left\{\tau_{n, 3}\right\}$, and increases by two when $\tau$ crosses $\left\{\tau_{n, 4}\right\}$. With this notation, the coexistence equilibrium is stabilized by dispersal and travel time delay when $\tau_{0,3}<\tau<\tau_{0,1}$. For the intermediate case $\frac{\epsilon A}{2}<d<\epsilon A$, the analysis proceeds similarly to the case for large $d$. We omit the details.

The results from this analysis are summarized in Table 3.1 and illustrated in Figures 1 and 2. The solid and starred curves in this figure correspond to $\tau_{n, 1}$ and $\tau_{n, 2}$; dotted and dashed curves depict $\tau_{n, 3}$ and $\tau_{n, 4}$. The number of solutions with positive real part (counting multiplicity) decreases by two when $\tau$ increases and passes through dotted and starred curves, and increases by two when $\tau$ crosses solid and dashed curves. We observe one region in the $(d, \tau)$-plane where the coexistence steady state is unstable for a single patch but stable in the coupled two-patch system. We illustrate the time course of a typical solution in this region in Figure 2. The densities exhibit decaying oscillations and eventually stabilize. 


\begin{tabular}{|c|c|c||c|}
\hline & $0<d<\epsilon A / 2$ & $d>\epsilon A / 2$ & single patch \\
\hline$A<0$ & stable & stable & stable \\
\hline$A>0$ & unstable & stable if $\tau_{0,3}<\tau<\tau_{0,1}$ & unstable \\
\hline
\end{tabular}

Table 2: Summary of the stability analysis of the coexistence equilibrium with $\alpha=0$

Reverting to dimensional variables and interpret these stability results in the light of condition (3.8). The first condition for the existence of the coexistence state $\left(h^{*}>\right.$ $0)$ is $m<a$, or $1 / m>1 / a$. The mean life expectancy for the predator has to be larger than the mean time to capture. The condition $A>0$ translates into a being much larger than $m$, or $1 / m \gg 1 / a$. The term in brackets in (3.8) is bounded by unity. The condition $1 / a \ll 1 / m$ then implies that inequality (3.8) is violated if $D \gg r / 2$ or $1 / D \ll 2 / r$. Hence, if the mean time spent in a patch $(1 / D)$, is much shorter than the time scale of reproduction, then an unstable coexistence can be stabilized by dispersal (with delay) between two patches. Stabilization can occur when $\tau$ is on the order of unity, i.e. when the travel time delay is of the same order as the mean time to capture.

\subsection{Predator-dependent dispersal $(\alpha>0)$}

When prey dispersal depends on perceived predation risk, a new phenomenon occurs, namely dispersal may destabilize an otherwise stable coexistence state. This result follows from a detailed analysis of the characteristic equations (3.2) along the lines of the previous section and Section 5 using again the results by Cooke and Grossman (1982). As in the previous section, zero is not an eigenvalue, and we need to understand when purely imaginary roots of these equations occur. After lengthy calculations, we find that such roots occur if and only if the following inequality holds:

$$
2 B \epsilon+2 d\left[\alpha \gamma B+\epsilon A\left(\frac{\alpha \gamma}{\mu} B+(1-\alpha)\right)\right]>2 B \sqrt{\epsilon^{2}+2 d \alpha \gamma \epsilon}+\epsilon^{2} A^{2} .
$$

Since the term on the right is greater than $2 B \epsilon$, this inequality cannot be satisfied if the term in square brackets is negative, i.e. $\alpha \gamma B+\epsilon A\left(\frac{\alpha \gamma}{\mu} B+(1-\alpha)\right) \leq 0$. Hence, we 


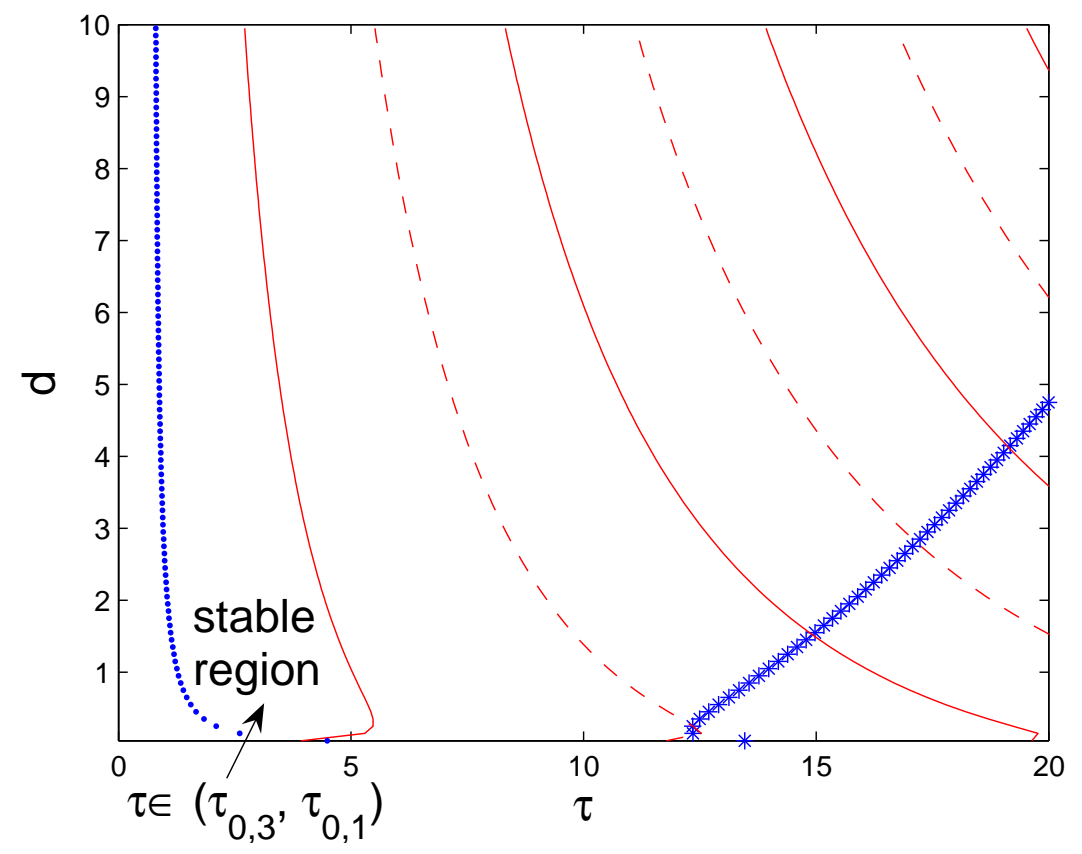

Figure 1: Stability region for density-independent dispersal and $A>0$. For any fixed $d>0$, the coexistence equilibrium is unstable when $\tau=0$. As $\tau$ increases and crosses the dotted curve that corresponds to $\tau_{n, 3}$, the coexistence steady state is stabilized. As $\tau$ increases further, it crosses the solid line corresponding to $\tau_{n, 1}$, so that the coexistence equilibrium becomes unstable again. As $\tau$ increases and passes through a solid $\left(\tau_{n, 1}\right)$ curve or a dashed $\left(\tau_{n, 2}\right)$ curve, two eigenvalues with positive real part appear. When $\tau$ passes through a dotted $\left(\tau_{n, 3}\right)$ or starred $\left(\tau_{n, 4}\right)$ curve, two eigenvalues with positive real part disappear. In the figure, there is only one stability region, but if parameters were chosen such that the starred curve were shifted to the left, another region of stability would appear. Parameter values are $\epsilon=1, k=2$, and $\mu=0.2$. 


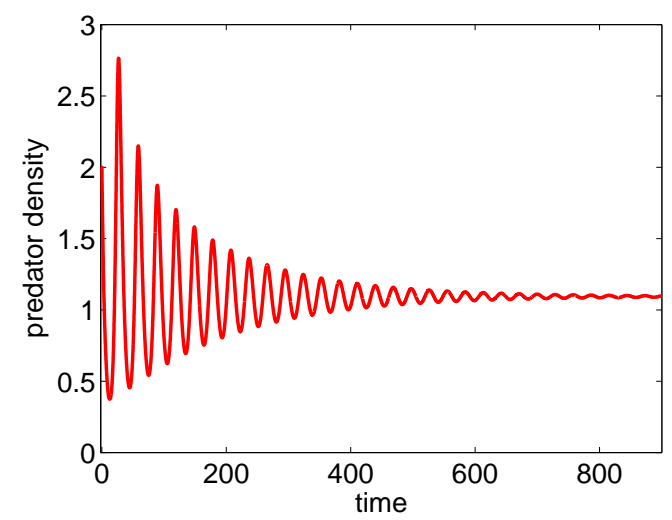

Figure 2: Density-independent dispersal with travel time delay stabilizes the populations at the positive coexistence equilibrium. Parameters are as in the previous figure so that $A=0.05>0$, and dispersal parameters are chosen in the stable region as $d=1, \tau=2$. Only the predator density on one patch is plotted. Densities on the other patch are indistinguishable after a short initial transient; prey densities in both patches follow the same pattern. In particular, the densities between patches are in synchrony. Stabilization occurs also for smaller values of $\tau$, for example with $\epsilon=0.8, \tau=1$ and $d=4$. The approach to equilibrium is then very slow (plot not shown). 
get a negative upper bound for $A$ that guarantees stability of the two-patch system

$$
A \leq A^{*}(\alpha, \gamma):=-\frac{\alpha \gamma B}{\epsilon\left(\frac{\alpha \gamma}{\mu} B+(1-\alpha)\right)}<0 .
$$

Then there is a window of opportunity for $A^{*}<A<0$ where the coexistence state is stable in the absence of dispersal but could be destabilized by dispersal. We investigate this possibility in more detail.

The threshold value $A^{*}(\alpha, \gamma)$ is strictly decreasing in both arguments, and $A(0,0)=$ 0 . Hence, as the proportion $(\alpha)$ of predator-induced dispersal or its strength $(\gamma)$ increase, the interval $A^{*}<A<0$ of potential destabilization becomes larger. For each $A^{*}<A<0$, there is a critical dispersal rate $d^{*}$ for which inequality (3.9) becomes an equality. We can calculate

$$
d^{*}=\frac{\epsilon^{2} A^{2} \alpha \gamma B+\left[\frac{\alpha \gamma}{\mu} B+(1-\alpha)\right]\left(\epsilon^{3} A^{3}-2 \epsilon^{2} A B\right)+\sqrt{\Delta}}{2\left[\alpha \gamma B+\epsilon A\left(\frac{\alpha \gamma}{\mu} B+(1-\alpha)\right)\right]^{2}},
$$

where

$$
\begin{aligned}
\Delta= & 4 B^{2} \epsilon^{2}\left\{\left[\alpha \gamma B+\epsilon A\left(\frac{\alpha \gamma}{\mu} B+(1-\alpha)\right)\right]^{2}+B^{2} \alpha^{2} \gamma^{2}\right. \\
& \left.-\alpha \gamma\left(2 B-\epsilon A^{2}\right)\left[\alpha \gamma B+\epsilon A\left(\frac{\alpha \gamma}{\mu} B+(1-\alpha)\right)\right]\right\} .
\end{aligned}
$$

All else being equal, this threshold value increases with $\alpha$, see Figure 3.

With these thresholds for $A$ and $d$, we have several cases to consider, see Table 3. When $A<A^{*}$ the coexistence state is stable independent of dispersal. When $A^{*}<A<0$ and $0 \leq d<d^{*}$ the coexistence equilibrium is still stable. However, for $A^{*}<A<0$ and $d>d^{*}$ dispersal may destabilize the stable coexistence state for the single patch. A similar analysis as in the previous section (for more details, see Section 5 in Cooke and Grossman (1982)) gives a critical value $\tau^{*}$ such that coexistence is stable for all $\tau \in\left[0, \tau^{*}\right)$ but becomes unstable when the delay $\tau$ is larger. We illustrate this destabilizing effect in Figure 4. We choose parameter values such that $A^{*}<A<0$. Then the system is stable without dispersal, but unstable with density-dependent dispersal, as the densities in Figure 4 show.

Finally, when $A>0$, the situation is similar to Case 2 in the previous section, but explicit calculations are more cumbersome since they include additional terms 


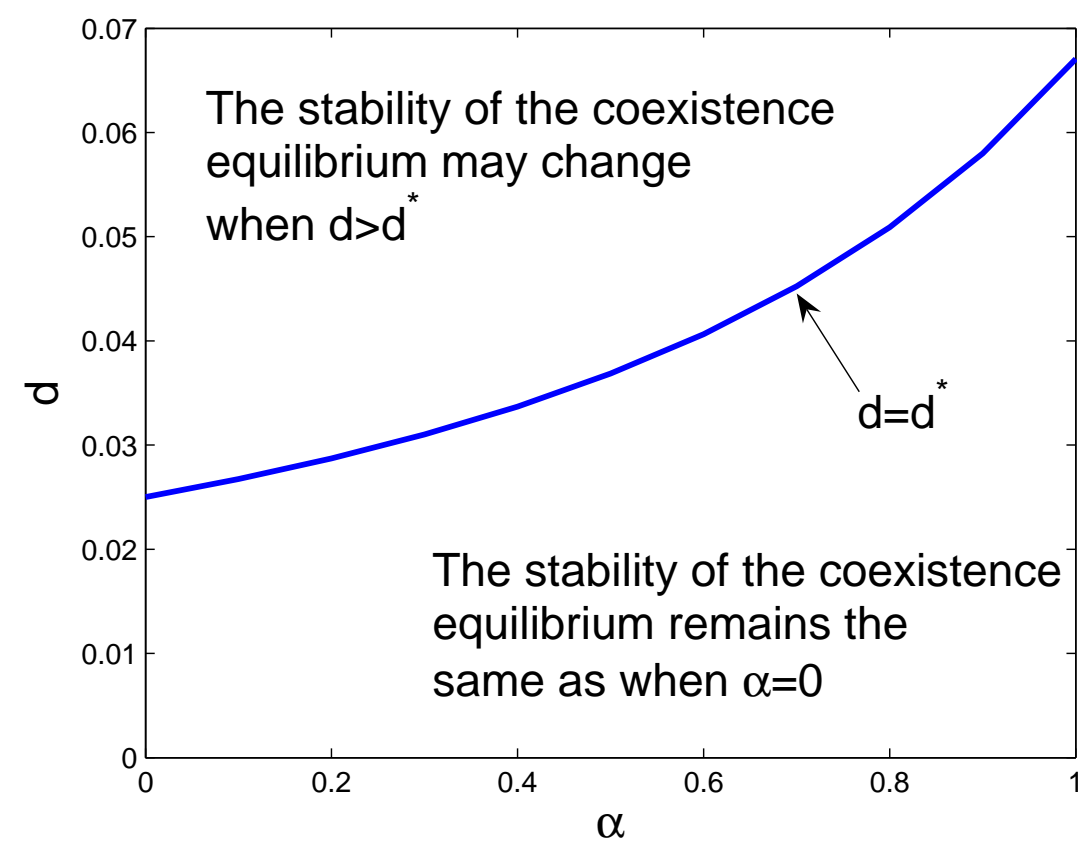

Figure 3: The critical dispersal rate $d^{*}$ is an increasing function of $\alpha$, the strength of density-dependent dispersal. When $d<d^{*}$ the stability of the coexistence equilibrium is the same as for $\alpha=0$. When $d>d^{*}$, the stability of the coexistence state can change, compared to the case with $\alpha=0$, provided that the travel time delay, $\tau$, is in the appropriate range (see text for details on that range). Parameters are $\epsilon=1$, $\gamma=0.5, k=2, \mu=0.2$. These choices lead to $A=-1 / 20>A^{*}$. 


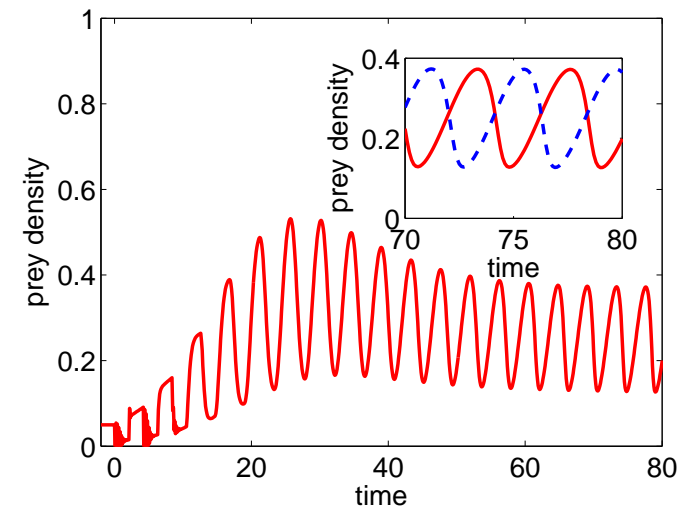

(a) Prey density.

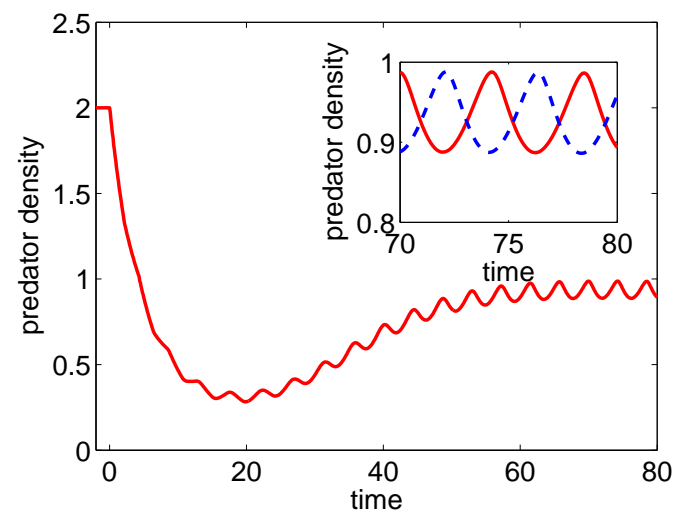

(b) Predator density.

Figure 4: Sustained oscillations can arise from the interplay between densitydependent dispersal and travel time delay. The coexistence equilibrium of the twopatch system is unstable even though the coexistence state is stable on each patch in the absence of dispersal. The densities on the two patches oscillate out of phase. The large plots show the respective densities on one of the patches, the insets show the corresponding densities on patch 1 (solid) and patch 2 (dashed). Parameter values are $\epsilon=1, \gamma=0.5, \mu=0.2, k=1, \alpha=0.9, \tau=2$, and $d=20$. Accordingly, we calculate $A^{*}=-0.1459<A=-0.1<0$, see text for details. 
with $\alpha$. More specifically, one can define $\bar{\tau}_{n, i}, i=1,2,3,4$ that act in the same way as $\tau_{n, i}, i=1,2,3,4$ in the previous section. Specifically, the system can be stabilized when $d>d^{*}$ and $\tau \in\left(\bar{\tau}_{0,3}, \bar{\tau}_{0,1}\right)$. In Figure 5 we compare the region in the $(d, \tau)$-plane where stabilization happens for different values of $\alpha$. The case $\alpha=0$ is the same as in Figure 1. For $\alpha=1$, the stability region is much narrower with respect to $\tau$, at least for small $d$.

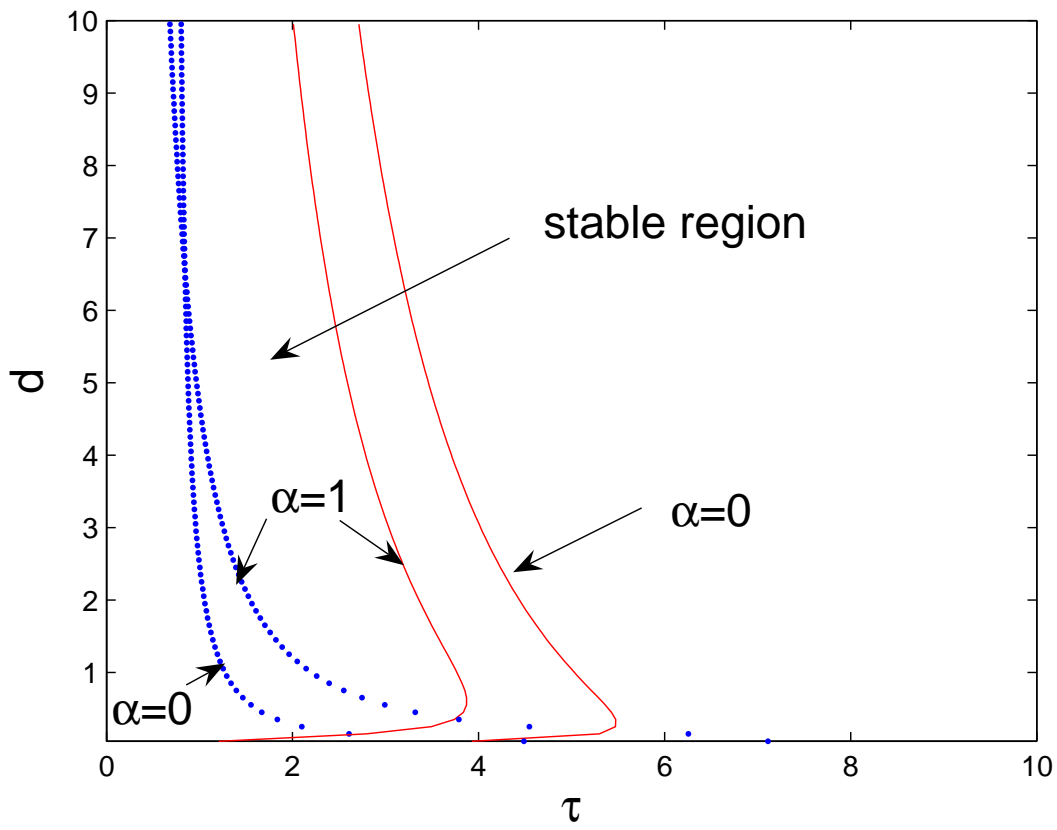

Figure 5: Illustration of how the stability region for $A>0$ in the $d$ - $\tau$-plane changes between density-independent $(\alpha=0)$ and density-dependent $(\alpha=1)$ dispersal. The dotted and solid curves for $\alpha=0$ are the same as the curves bounding the stability region in Figure 1. The range of $\tau$-values for which stabilization occurs decreases significantly, at least for small enough values of $d$. Parameter values are the same as in Figure 1.

\section{Discussion}

The local conditions and mechanisms that affect the stability of a community have been studied intensely since the work by Rosenzweig and MacArthur (1963) and May 


\begin{tabular}{|c|c|c|}
\hline & $0<d<d^{*}$ & $d>d^{*}$ \\
\hline$A<A^{*}$ & stable & stable \\
\hline$A^{*}<A<0$ & stable & stable if $0<\tau<\tau^{*}$ \\
\hline$A>0$ & unstable if $d<\min \left(\epsilon A / 2, d^{*}\right)$ and otherwise stable if $\bar{\tau}_{0,3}<\tau<\bar{\tau}_{0,1}$ \\
\hline
\end{tabular}

Table 3: Summary of the stability analysis of the coexistence equilibrium with $\alpha \in$ $(0,1]$

(1973), and are by now fairly well understood. Our understanding of the effects of movement in spatially coupled communities on their stability is much less developed, but some principles have emerged in recent years. Movement between identical patches, either as density-independent dispersal (Jansen, 1995) or as a densitydependent behavioral response (Hauzy et al., 2010), does not change the stability of the community on a single patch, as long as movement is instantaneous. Including movement time across a landscape in simple density-independent dispersal models may stabilize local populations, but never destabilizes local stable communities (Neubert et al., 2002; Klepac et al., 2007). We studied how density-dependent and independent dispersal and movement time interact to affect community stability.

We used a two-patch Rosenzweig-MacArthur model with prey-only dispersal. For density-independent dispersal, our results agree with and extend the previous results that including travel time acts as a stabilizing mechanism. Our main result is that the interplay between density-dependent dispersal and travel time between patches can destabilize the community even though neither of these mechanisms is destabilizing on its own. Our analysis also reveals that temporal scales over which this interplay operates are compatible with temporal scales over which travel time and density-dependent movement are expected to overlap in some natural systems. Since our analysis relies on local stability techniques, it is independent of the specific form of the functions chosen to model growth, consumption and density-dependent dispersal. The overall results should hold more generally, as long as the general shape of the functions is similar, in particular, density-dependent movement decreases with 
conspecific density and increases with predator density.

\section{Landscape heterogeneity and between-patch movement time}

Landscape heterogeneity and geometry are crucial determinants of individual movement. Movement in heterogeneous landscapes is often idealized as individuals dispersing among focal patches where demographic processes take place (Goodwin and Fahrig, 2002; Nystrand et al., 2010; Strevens and Bonsall, 2011). Empirically, landscape matrix surrounding focal patches does affect movement patterns (Ricketts, 2001) and travel time (Bélisle, 2005) between patches. While some more recent theories have explored the effects of heterogeneous movement rates from patches (Hanski and Gilpin, 1991; Hanski, 2001), very few have explicitly included travel time between patches (Neubert et al., 2002; Klepac et al., 2007).

Often, travel time can be assumed to be much faster than demographic processes, thus supporting the assumption of instantaneous dispersal found in most theories. However, even arbitrarily short delays in dispersal can have very strong impacts on predicted synchrony of predator-prey oscillations between patches (Wall et al., 2013). Instantaneous and random dispersal has no direct stabilizing effect on an unstable equilibrium (Briggs and Hoopes, 2004). Instead, low movement rate can only stabilize the metacommunity by maintaining asynchronous dynamics between patches (Taylor et al., 1990). Our analytical study agrees with, and generalizes previous numerical analyses (Klepac et al., 2007) showing a direct stabilizing effect of dispersal in the presence of delayed dispersal. Our analysis reveals how travel time and the time scale of movement can interact to stabilize oscillations. We show that intermediate travel time on the order of time to capture by the predator can actually allow strong dispersal rates to stabilize the otherwise unstable 2-patch system (Figure 2). In systems with sedentary predators and mobile prey, frequent prey movement and travel time approaching their life span, provide sufficient decoupling between predator and prey densities to stabilize the equilibrium coexistence of the metacommunity. This prediction applies to prey with long-lived dispersing phases such as aquatic and marine species with long pelagic larval stage, interacting with sedentary predators that can 
have a much shorter or no pelagic larval phase (Kinlan and Gaines, 2003; Kinlan et al., 2005)).

\section{Density-dependent dispersal and time scales of movement}

The many mechanisms and causes of movement and dispersal are typically greatly simplified in ecological models and implemented as either density independent or density dependent rates. Multiple mechanisms of animal movement are necessarily associated with multiple temporal scales (Nieminen, 1996; Goodwin and Fahrig, 2002) that can conflict with the simplicity of spatially-explicit models (Cantrell and Cosner, 1996; Auger et al., 2008). Predator-prey models based on demographic processes typically assume instantaneous and density-independent movement (Jansen, 2001) on the same time scale are demographics. In contrast, predator avoidance or foraging behavior can trigger density-dependent movement on much faster time scales (Abdllaoui et al., 2007; Hauzy et al., 2007). In particular, the time to movement from a patch can be comparable to travel time between patches. Our results show that important dynamical phenomena emerge from integrating fast density-dependent trigger of movement with travel time over similar temporal scales (see Table 3). For example, frequent prey movement driven by density-dependent response to predator density can destabilize the metacommunity equilibrium if travel time is sufficiently close to the time to capture (see Figure 4).

One important insight emerging from this result is that frequent movement is an important driver of stability when it is driven by a behavioral mechanism (predator avoidance), which makes the prediction broadly applicable to ecological systems. Even more importantly, our analysis shows how metacommunities can be destabilized by the interplay between several common properties (prey movement, predator avoidance and travel time) when none of these properties is destabilizing on its own (Hauzy et al., 2010). Our specific predictions suggest that such destabilization could be observed in systems where frequent prey movement in response to predators is associated with long travel time. The effects of density-dependent dispersal and travel time are not limited to community stability, they also extend to the dynamics 
of nonequilibrium communities (Ramanantoanina et al., 2011). Hauzy et al. (2010) demonstrate that density-dependent dispersal can shift periodic to chaotic behavior. Travel time for density-independent dispersal can lead to a shift from in-phase to anti-phase locking in cyclic communities (Wall et al., 2013). The behavior observed in Figure 4 points to a stable, anti-phase locked situation. In future work, we shall explore the effect of density-dependent dispersal with travel time delay on the stability of the phase-locked solution and potential switches.

\section{Future challenges}

Our study predicts that movement driven by predator avoidance and travel time between habitats can destabilize metacommunities. Our model still includes relatively simplistic assumptions, for example, we only included travel time between patches. In reality, travel through unfavorable regions could induce mortality, particularly if dispersal is stress-induced (predator avoidance). Linking mechanisms on lower scales, one could derive expressions for travel time between patches and dispersal-induced mortality from multi-scale models for individual movement in continuous space (Cobbold and Lutscher, 2014). Another avenue for future research is to include behaviorbased dispersal of the predator as well. Specifically, the dispersal rate of the predator should decrease as a function of prey density. Some work in this direction was already started, when the prey movement rate depends on predator density alone (Abdllaoui et al., 2007; Mchich et al., 2007, 2005). Despite its limitations, our study contributes to the integration of complex effects of landscapes and behavior on movement, to a general theory of metacommunity stability. As our study suggests, this integration

and its application to natural systems, depend on the explicit assessment of temporal and spatial scales associated with natural landscapes and life histories of species that control movement.

\section{Acknowledgments}

YZ is partly supported by the Centre de Recherches Mathématiques (Montréal) through the pan-Canadian thematic year "Model and methods in ecology, epidemiol- 
ogy and public health (2013)". FL gratefully acknowledges funding from the Natural Science and Engineering Research Council of Canada (NSERC). FG acknowledges support from NSERC through the Canadian Healthy Oceans network (CHONe).

\section{Appendix A: Stability of the coexistence steady state when $\tau=0$}

In this appendix, we demonstrate that the coexistence steady state for the two-patch model (2.2) with $\tau=0$ is stable if and only if the coexistence state is stable for the single-patch model (2.3). The transformation introduced in Hauzy et al. (2010) gives a block-diagonal matrix whose stability properties can easily be determined.

We denote the vector of densities on patch $i$ with $X_{i}=\left(h_{i}, p_{i}\right)^{T}$. Then system (2.2) with $\tau=0$ can be written as

$$
\begin{aligned}
& \frac{d X_{1}}{d s}=F\left(X_{1}\right)-G\left(X_{1}\right)+G\left(X_{2}\right) \\
& \frac{d X_{2}}{d s}=F\left(X_{2}\right)-G\left(X_{2}\right)+G\left(X_{1}\right)
\end{aligned}
$$

where

$$
\begin{aligned}
F\left(X_{i}\right) & =\left(\epsilon\left(h_{i}\left(1-\frac{h_{i}}{k}\right)-\frac{h_{i} p_{i}}{1+h_{i}}\right), \frac{h_{i} p_{i}}{1+h_{i}}-\mu p_{i}\right) \\
G\left(X_{i}\right) & =\left(d\left(\alpha \gamma \frac{p_{i} h_{i}}{1+h_{i}}+(1-\alpha) h_{i}\right), 0\right) .
\end{aligned}
$$

The crucial transformation consists of the change of variables: $U=\left(X_{1}+X_{2}\right) / 2$, $V=\left(X_{1}-X_{2}\right) / 2$. Then system (4.1) is equivalent to

$$
\begin{aligned}
\frac{d U}{d s} & =\frac{F(U+V)+F(U-V)}{2}, \\
\frac{d V}{d s} & =\frac{F(U+V)-F(U-V)}{2}+G(U-V)-G(U+V) .
\end{aligned}
$$

Linearizing system (4.2) at the coexistence state $U^{*}=\left(h^{*}, p^{*}\right)^{T}, V^{*}=(0,0)^{T}$, we 
get the linear system

$$
\begin{aligned}
& \frac{d U}{d s}=D F\left(U^{*}\right) U \\
& \frac{d V}{d s}=\left[D F\left(U^{*}\right)-2 D G\left(U^{*}\right)\right] V
\end{aligned}
$$

where $D F(\cdot)$ and $D G(\cdot)$ denote the Jacobian matrix of functions $F$ and $G$, respectively. This system is in block-diagonal form, and the steady state is stable if all eigenvalues of the two matrices $D F\left(U^{*}\right)$ and $D F\left(U^{*}\right)-2 D G\left(U^{*}\right)$ have negative real parts.

We calculate both matrices.

$$
D F\left(U^{*}\right)=\left(\begin{array}{cc}
\epsilon\left(1-2 h^{*} / k-p^{*} /\left(1+h^{*}\right)^{2}\right) & -\epsilon \mu \\
p^{*} /\left(1+h^{*}\right)^{2} & 0
\end{array}\right)
$$

and $D F\left(U^{*}\right)-2 D G\left(U^{*}\right)=$

$$
\left(\begin{array}{cc}
\epsilon\left(1-2 h^{*} / k-p^{*} /\left(1+h^{*}\right)^{2}\right)-2 d\left(\alpha \gamma p^{*} /\left(1+h^{*}\right)^{2}+(1-\alpha)\right) & -(\epsilon+2 d \alpha \gamma) \mu \\
p^{*} /\left(1+h^{*}\right)^{2} & 0
\end{array}\right) .
$$

Since the determinants of both matrices are positive, stability depends on the sign of the two traces. Since the trace of the second matrix is smaller than the one of the first, the coexistence state is stable precisely when the trace of $D F\left(U^{*}\right)$ is negative. But the matrix $D F\left(U^{*}\right)$ is precisely the community matrix of the single-patch MacArthurRosenzweig model (2.3).

The same method gives the additional result that $(k, 0, k, 0)$ is stable for $(2.2)$ if and only if $(k, 0)$ is stable for the single-patch system. That is, the local stability of symmetric equilibria does not depend on the values of $d$ and $\alpha$.

\section{Appendix B: Zeros of a transcendental polynomial}

A key element in our analysis is the control of eigenvalues with positive real part of the linearization at the coexistence state. The following Lemma from Cooke and Grossman (1982) shows that in our case, an eigenvalue with positive real part can 
only appear by passing through the imaginary axis and not, for example, in a saddlenode bifurcation. For ease of referral and convenience of the reader, we state this not-so-well known Lemma here.

Lemma 4.1 (Cooke and Grossman (1982)). Let $f(\lambda, \tau)=\lambda^{2}+a_{1} \lambda+a_{2} \lambda e^{-\tau \lambda}+$ $a_{3}+a_{4} e^{-\lambda \tau}$, where $a_{i}, \tau$ are real numbers and $\tau \geq 0$. Then, as $\tau$ varies, the sum of the multiplicities of zeros of $f$ in the open right half-plane can change only if a zero appears on or crosses the imaginary axis.

\section{References}

Abdllaoui, A. E., Auger, P., Kooi, B. W., De la Parra, R. B., and Mchich, R. (2007). Effects of density-dependent migrations on stability of a two-patch predator-prey model. Mathematical biosciences, 210(1), 335-354.

Abrams, P. and Ruokolainen, L. (2011). How does adaptive consumer movement affect population dynamics in consumer resource metacommunities with homogeneous patches? Journal of Theoretical Biology, 277, 99-110.

Abrams, P. A. (2007). Habitat choice in predator-prey systems: spatial instability due to interacting adaptive movements. The American Naturalist, 169(5), 581-594.

Auger, P., de La Parra, R. B., Poggiale, J.-C., Sánchez, E., and Sanz, L. (2008). Aggregation methods in dynamical systems and applications in population and community dynamics. Physics of Life Reviews, 5(2), 79-105.

Bélisle, M. (2005). Measuring landscape connectivity: the challenge of behavioral landscape ecology. Ecology, 86(8), 1988-1995.

Briggs, C. J. and Hoopes, M. F. (2004). Stabilizing effects in spatial parasitoidhost and predator-prey models: a review. Theoretical population biology, 65(3), $299-315$. 
Cantrell, R. and Cosner, C. (1996). Models for predator-prey systems at multiple scales. Siam Review, 38(2), 256-286.

Chakraborty, A., Singh, M., Lucy, D., and Ridland, P. (2007). Predator-prey model with prey taxis and diffusion. Mathematical and computer modelling, 46, 482-498.

Cobbold, C. and Lutscher, F. (2014). Mean occupancy time: linking mechanistic movement models, population dynamics and landscape ecology to population persistence. J. Math. Biol., 68, 549-579.

Cooke, K. L. and Grossman, Z. (1982). Discrete delay, distributed delay and stability switches. Journal of Mathematical Analysis and Applications, 86(2), 592-627.

Cuddington, K. and Yodzis, P. (2002). Predator-prey dynamics and movement in fractal environments. The American Naturalist, 160, 119-134.

Goodwin, B. J. and Fahrig, L. (2002). Effect of landscape structure on the movement behaviour of a specialized goldenrod beetle, trirhabda borealis. Canadian Journal of Zoology, 80(1), 24-35.

Hanski, I. (2001). Spatially realistic theory of metapopulation ecology. Naturwissenschaften, 88(9), 372-381.

Hanski, I. and Gilpin, M. (1991). Metapopulation dynamics: brief history and conceptual domain. Biological journal of the Linnean Society, 42(1-2), 3-16.

Hauzy, C., Hulot, F. D., Gins, A., and Loreau, M. (2007). Intra-and interspecific density-dependent dispersal in an aquatic prey-predator system. Journal of animal ecology, 76(3), 552-558.

Hauzy, C., Gauduchon, M., Hulot, F. D., and Loreau, M. (2010). Density-dependent dispersal and relative dispersal affect the stability of predator-prey metacommunities. Journal of theoretical biology, 266(3), 458-469.

Jansen, V. A. (1995). Regulation of predator-prey systems through spatial interactions: a possible solution to the paradox of enrichment. Oikos, pages 384-390. 
Jansen, V. A. (2001). The dynamics of two diffusively coupled predator-prey populations. Theoretical Population Biology, 59(2), 119-131.

Kinlan, B. P. and Gaines, S. D. (2003). Propagule dispersal in marine and terrestrial environments: a community perspective. Ecology, 84(8), 2007-2020.

Kinlan, B. P., Gaines, S. D., and Lester, S. E. (2005). Propagule dispersal and the scales of marine community process. Diversity and distributions, 11(2), 139-148.

Klepac, P., Neubert, M. G., and van den Driessche, P. (2007). Dispersal delays, predator-prey stability, and the paradox of enrichment. Theoretical population biology, 71(4), 436-444.

Kot, M. (2001). Elements of Mathematical Ecology. Cambridge Univ. Press, Cambridge.

May, R. M. (1973). Stability and complexity in model ecosystems. Princeton University Press, Princeton, New Jersey.

McCauley, E., Wilson, W., and de Roos, A. (1993). Dynamics of age-structures predator-prey interations: Individual based models and population level formulations. The American Naturalist, 142, 412-442.

Mchich, R., Bergam, A., and Raïssi, N. (2005). Effects of density dependent migrations on the dynamics of a predator prey model. Acta biotheoretica, 53(4), $331-340$.

Mchich, R., Auger, P., and Poggiale, J.-C. (2007). Effect of predator density dependent dispersal of prey on stability of a predator-prey system. Mathematical biosciences, 206(2), 343-356.

Nathan, R. and Giuggioli, L. (2013). A milestone for movement ecology research. Movement Ecology, 1, 1. 
Neubert, M. G., Klepac, P., and Van den Driessche, P. (2002). Stabilizing dispersal delays in predator-prey metapopulation models. Theoretical population biology, 61(3), 339-347.

Nieminen, M. (1996). Migration of moth species in a network of small islands. Oecologia, 108(4), 643-651.

Nystrand, M., Griesser, M., Eggers, S., and Ekman, J. (2010). Habitat-specific demography and source-sink dynamics in a population of siberian jays. Journal of animal ecology, 79(1), 266-274.

Ramanantoanina, A., Hui, C., and Ouhinou, A. (2011). Effects of density-dependent dispersal behaviours on the speed and spatial patterns of range expansion in predator-prey metapopulations. Ecological Modelling, 222(19), 3524-3530.

Ricketts, T. H. (2001). The matrix matters: effective isolation in fragmented landscapes. The American Naturalist, 158(1), 87-99.

Rosenzweig, M. L. and MacArthur, R. H. (1963). Graphical representation and stability conditions of predator-prey interactions. American Naturalist, 97(895), 209.

Ruokolainen, L., Abrams, P., McCann, K., and Shuter, B. (2011). The roles of spatial heterogeneity and adaptive movement in stabilizing (or destabilizing) simple metacommunities. Journal of Theoretical Biology, 291, 76-87.

Sapoukhina, N., Tyutyunov, Y., and Arditi, R. (2003). The role of prey taxis in biological control: A spatial theoretical model. The American Naturalist, 162, $61-76$.

Strevens, C. M. and Bonsall, M. B. (2011). Density-dependent population dynamics and dispersal in heterogeneous metapopulations. Journal of animal ecology, 80(1), 282-293.

Taylor, A. D. et al. (1990). Metapopulations, dispersal, and predator-prey dynamics: an overview. Ecology, 71(2), 429-433. 
Wall, E., Guichard, F., and Humphries, A. R. (2013). Synchronization in ecological systems by weak dispersal coupling with time delay. Theoretical Ecology, 6(4), 405-418. 